\title{
A pilot study of biomechanical assessment before and after an integrative training program for adolescents with juvenile fibromyalgia
}

Susan T. Tran', Staci Thomas², Christopher DiCesare ${ }^{2}$, Megan Pfeiffer ${ }^{3}$, Soumitri Sil $^{4}$, Tracy V. Ting ${ }^{5,6}$, Sara E. Williams ${ }^{3,6}$, Gregory D. Myer $r^{2,6,7,8,9}$ and Susmita Kashikar-Zuck ${ }^{3,6^{*}}$ (i)

\begin{abstract}
Background: Adolescents with juvenile fibromyalgia (JFM) tend to be very sedentary and avoid participation in physical activity. A prior study suggested that JFM patients show altered biomechanics compared to healthy adolescents which may make them more prone to pain/injury during exercise. A new intervention combining well established cognitive behavioral therapy (CBT) techniques with specialized neuromuscular exercise —Fibromyalgia Integrative Training for Teens (FIT Teens) was developed and shown to be promising in improving functioning in adolescents with JFM. In contrast to traditional exercise programs such as aerobic or resistance training, neuromuscular training is a tailored approach which targets gait, posture, balance and movement mechanics which form the foundation for safe exercise participation with reduced risk for injury or pain (and hence more tolerable by JFM patients). The aim of this pilot feasibility study was to establish whether objective biomechanical assessment including sophisticated 3-D motion analysis would be useful in measuring improvements in strength, balance, gait, and functional performance after participation in the 8-week FIT Teens program.

Methods: Eleven female participants with JFM (ages 12-18 years) completed pre- and post-treatment assessments of biomechanics, including walking gait analysis, lower extremity strength assessment, functional performance, and dynamic postural stability.

Results: Descriptive data indicated that mechanics of walking gait and functional performance appeared to improve after treatment. Hip abduction strength and dynamic postural control also demonstrated improvements bilaterally.

Conclusions: Overall, the results of this pilot study offer initial evidence for the utility of biomechanical assessment to objectively demonstrate observable changes in biomechanical performance after an integrated training intervention for youth with JFM. If replicated in larger controlled studies, findings would suggest that through the FIT Teens intervention, adolescents with JFM can progress towards normalized strength and biomechanics, which may enhance their ability to engage in physical exercise.
\end{abstract}

Keywords: Juvenile fibromyalgia, Chronic pain, Biomechanical assessment, Physical activity, Adolescents

\footnotetext{
* Correspondence: susmita.kashikar-zuck@cchmc.org

${ }^{3}$ Division of Behavioral Medicine and Clinical Psychology- MLC 7039,

Cincinnati Children's Hospital Medical Center, 45229 Cincinnati, OH, USA

${ }^{6}$ Department of Pediatrics, University of Cincinnati, College of Medicine,

Cincinnati, OH, USA

Full list of author information is available at the end of the article
} 


\section{Background}

Juvenile fibromyalgia (JFM) is characterized by chronic widespread musculoskeletal pain, occurs in 2-6\% of children, primarily in adolescent girls [1-5], and is associated with significant physical and emotional impairment [6-11]. Typically, symptoms are best managed through a combination of medication, regular exercise, and cognitive-behavioral therapy (CBT) [12-14]. Although routine engagement in physical exercise is strongly recommended for pain management in musculoskeletal conditions such as JFM [12], adherence to exercise in patients with JFM is poor $[15,16]$ and the majority of individuals with fibromyalgia are sedentary [17-19]. Prolonged sedentary behavior may contribute to physical deconditioning and a loss of confidence in one's ability to participate in physical activity [20], which reinforces the cycle of pain and disability.

Maintaining adequate daily physical activity is a challenge for patients with JFM because exercise may be difficult or uncomfortable, particularly after a pain flare [21]. Our recent investigation of adolescents with JFM using sophisticated biomechanical analyses documented altered biomechanics, deficits in functional performance, lower strength, and greater fear of movement compared to healthy peers [22]. These altered movement patterns are similar to those that increase risk of injury [23, 24]. It is likely that poor body biomechanics may be a key contributor to patients' high fear of movement [22] and difficulty adhering to traditional aerobic or resistance training programs. Traditional aerobic or resistance training programs, even those with a graded approach, do not focus on establishing fundamental movement competence prior to implementing the recommended exercises. Evidence from sports medicine and injury prevention research supports the importance of developing core strength, conditioning, and basic movement skills prior to engagement in higher levels of physical activity $[25,26]$.

Tailored exercise programs are needed for fibromyalgia patients [27] to improve their movement competence while simultaneously enhancing psychological confidence in engaging in physical exercise. CBT has been shown to effectively decrease functional disability and depressive symptoms in patients with JFM [28]; however, on its own, CBT did not increase engagement in vigorous physical activity [29]. Integrating behavioral coping skills training, including targeting maladaptive thought patterns such as fear of pain, with a tailored exercise program could have the potential to optimize pain and disability outcomes in JFM.

The Fibromyalgia Integrative Training for Teens (FIT Teens) intervention was created by modifying a specialized integrative neuromuscular exercise program used in injury prevention for young athletes for the needs of adolescents with JFM. FIT Teens incorporates CBT strategies to allow participants to apply pain coping skills in-vivo as they learn the neuromuscular exercises. A qualitative investigation documented the feasibility, tolerability, and initial efficacy of the new FIT Teens program for patients with JFM [30]. Patients reported high levels of engagement, felt they were coping more effectively, experiencing less fatigue, and becoming more active after treatment. Participants' self-reports on validated measures also reflected improvements in functional disability, depression, fear of movement, pain catastrophizing, and readiness to change [31]. Measurement of objective physical improvements would help to more fully capture the effects of the FIT Teens intervention (beyond self-report) and also better understand the mechanisms of improved physical function. Unfortunately, despite the availability of sophisticated new technologies for assessing gait and functional movements there is very little research in the assessment of movement biomechanics in patients with musculoskeletal pain, and none on adolescents with JFM. The focus of the current study was to examine whether objective biomechanical assessments showed utility and responsiveness to change after the 8-week FIT Teens Program - specifically on measures of gait mechanics, dynamic postural stability, functional performance, and knee and hip strength.

\section{Methods \\ Participants}

Youth (12-18 years old) were eligible if they were diagnosed with JFM by a pediatric rheumatologist or pain physician using Yunus and Masi criteria (Table 1) [5]. Additionally, only participants with moderate to high pain-related disability (Functional Disability Inventory score $\geq 12$ ) [32] were included. Participants were

Table 1 Yunus and Masi [5] diagnostic criteria for juvenile primary fibromyalgia syndrome

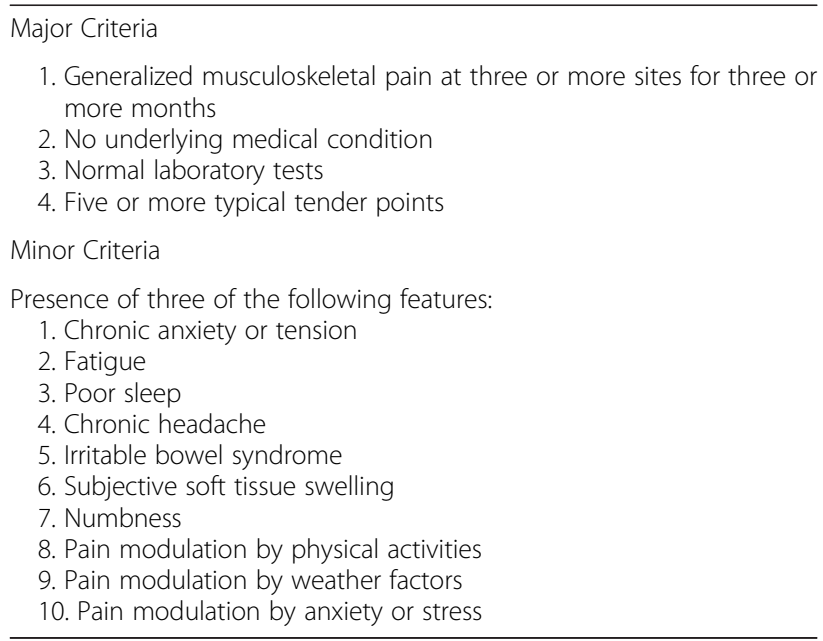


excluded if they had a diagnosis of a comorbid rheumatic disease (e.g., juvenile arthritis, systemic lupus erythematous), untreated major psychiatric diagnosis (e.g., major depression, bipolar disorder, and psychosis), documented developmental delay, any medical condition determined by their physician to be a contraindication for exercise, or ongoing participation in CBT for pain. For details, screening and recruitment procedures were described previously [30].

Research assistants identified potential participants from pediatric rheumatology and pain clinics at a large children's hospital. Physicians confirmed medical eligibility and introduced the study to patients and their caregivers. If interested, a research assistant met with families to discuss the study, answer any questions, and obtain written informed consent and assent. This study was approved by the Institutional Review Board of the Children's Hospital and the rights of participants were protected.

\section{Biomechanical testing procedures}

Prior to and after the intervention, participants completed biomechanical assessments with a trained exercise physiologist at the Children's Hospital's Sports Medicine Biodynamics \& Human Performance Laboratory. Participants completed measures that assessed walking gait, dynamic postural stability, lower extremity strength, and functional performance that included 3D motion capture technology. During the gait analysis and functional performance assessments, participants wore 43 retroreflective markers placed on standardized locations [22, 33], with a minimum of three markers per segment, including bilateral lower extremities (e.g., foot, shank, and thigh) and trunk (e.g., pelvis and thorax).

\section{Gait analysis}

Kinematics of walking have been shown to be dependent on gait speed [34], so walking gait was analyzed under two conditions: 1) self-selected pace and 2) standardized pace (1.2 meters/second) at $240 \mathrm{~Hz}$. For the self-selected pace condition, participants were instructed to walk at their normal pace and were given no corrective feedback. During the standardized pace condition, participants were given feedback to adjust their speed (either faster or slower) to standardize their pace by measuring the time it took to walk 3 meters. Normal gait speeds in healthy adults have been shown to range from 1.05 to $1.43 \mathrm{~m} / \mathrm{s}$ so the standardized pace was set to $1.2 \mathrm{~m} / \mathrm{s}$ $[35,36]$. Participants walked across the laboratory floor which was embedded with force plates (AMTI; Advanced Medical Technology, Inc., Watertown, MA) sampling at $1,200 \mathrm{~Hz}$, and three foot strikes on each side were recorded for each walking condition. Gait was captured using a 10-camera real-time high-speed 3-D motion analysis system (Raptor-E; Motion Analysis Corp., Santa Rosa, CA).

\section{Knee strength}

Isokinetic knee strength was assessed using the Biodex System II (Shirley, NY). Participants sat in the dynamometer chair secured with a thigh strap and waist strap aligning their hips at $90^{\circ}$ and aligning their knees with the rotation axis of the machine. The lower leg was strapped to the dynamometer arm 2 inches above the lateral malleolus. Participants practiced first, performing five continuous repetitions of maximal knee extension/ flexion starting at $90^{\circ}$ knee flexion; participants extended their knee to full extension and actively flexed the knee back to the starting position at an isokinetic speed of $300 \%$ second. After a period of rest, participants performed 10 continuous repetitions with maximal effort on each leg. Peak torques $(\mathrm{Nm} / \mathrm{kg})$ of knee extension and knee flexion were recorded [37].

\section{Hip strength}

Hip abduction strength was assessed using the Biodex System III. Participants stood facing the dynamometer head, aligning the center of their hip with the axis of rotation. The testing leg was strapped to the dynamometer arm just above the knee. Participants were instructed to kick out to the side with maximal effort five times and the testing speed was set to $120^{\circ}$ /second. Participants practiced first, rested, then performed five repetitions on each leg. Peak torque $(\mathrm{Nm} / \mathrm{kg})$ of each kick was recorded [38].

\section{Functional performance}

To perform the drop vertical jump task (DVJ), participants stood on a $31-\mathrm{cm}$ box with their feet shoulderwidth apart. They were instructed to drop off the box with both feet at the same time, land on the force plates in front of them, and immediately jump with both feet to perform a vertical jump with maximal effort towards an overhead target (a basketball). After watching the procedure demonstrated, participants completed practice trials (typically 2-3) until they were comfortable with the test. Participants were provided the opportunity for rest breaks after practicing and between trials; however, most participants did not require breaks. The 3-D motion analysis system was used to assess landing technique during the DVJ [39].

\section{Dynamic postural stability}

Adolescents with JFM often report difficulty with stability and balance and therefore a standardized tool used in prior studies [40-42] was used to assess postural stability. To perform the Star Excursion Balance Test, participants stood on one lower extremity at the center of the 
grid. After observing a demonstration of the task, participants were instructed to perform a maximal reach with the non-stance leg in the anterior, posteromedial, and posterolateral direction while maintaining balance on their single leg. The exercise physiologist visually measured the most distal reach distance of the foot in all three directions in centimeters. The process was then repeated while standing on the opposite extremity. Each participant performed at least two complete cycles for practice before the reach distances were recorded. Trials were repeated if the subject was unable to maintain their balance for the duration of the test, if weight was shifted to the reach leg, or if the heel of the stance leg did not maintain contact with the floor.

\section{Intervention}

The FIT Teens intervention was group-based (consisting of 3-4 participants per group) and conducted over eight weeks; participants met twice per week, 90 min per session ( 45 min each devoted to CBT and neuromuscular training). Groups met at the Children's Hospital's Sports Medicine Biodynamics \& Human Performance Laboratory, with the neuromuscular training taking place in a gym setting, and the CBT component in an adjoining conference room. The development and content of the FIT Teens program was fully described in a previous publication [30]. Briefly, the CBT components of the program were based on our published clinical trial [28] and consisted of pain coping skills training including pain education, relaxation skills, distraction, activity pacing, problem solving, and modifying negative and catastrophic thoughts about pain. Coping skills were modified and integrated into the neuromuscular training component in order to demonstrate in-vivo application of coping skills.

The resistive training protocol used in the neuromuscular training was specially designed to reduce incidence of muscle pain and minimize delayed-onset muscle soreness by gradually increasing the complexity of muscle actions. The protocol builds upon the foundations of movement by moving from most basic isometric "hold" exercises, to concentric "muscle shortening" exercises, to eccentric "muscle lengthening" exercises, and finally the full range of motion for "functional movement" (complete protocol published by Thomas et al. [43]). Given known deficits in biomechanics of youth with JFM, the program was tailored to each participant's baseline levels of ability and confidence in movement, and modified to allow participants to master each level before advancing. The final level is a culmination of all activities, built into functional movements that are relevant for activities of daily life. Additionally, considering that joint hypermobility is a relatively common occurrence in JFM [44], modifications were made as needed to protect joints from hyperextension. At the end of each session, participants were provided home practice instructions for coping skills (practiced every day) and physical exercises. Participants were instructed to practice neuromuscular exercises at home two days over the weekend in addition to the two sessions per week, exercising for a total of four times per week. During each session, homework diaries were reviewed to discuss practice and barriers to practice.

As described by Thomas et al. [43], the exercises included in the intervention were geared towards mimicking activities of daily living. Through each phase of the protocol, specific instructions were given to the participants to encourage proper muscle activation, and therefore optimum technique and body position. For example, the squat exercise was performed at each stage and careful instructions and feedback were given to encourage participants to activate not only their quadriceps, but also the posterior chain muscles (gluteus maximus, gluteus medius, etc.) to obtain and maintain good squat position by ensuring that the knees were not lined up anterior to the toes. In addition, knee alignment in the frontal plane was strongly emphasized such that the knees were not in a valgus position, or came in towards the midline of the body while in the squat position. Focus on knee and hip alignment encouraged activation of the hip abductors and external rotators. Training the muscles to perform in the correct position can assist in a more favorable landing position during functional movements like the DVJ.

\section{Data analysis}

All biomechanical kinematic measures were calculated relative to the participants' neutral (zero) alignment; measured by the participant standing still aligned with the laboratory coordinate system. All biomechanical data reduction was performed using custom Matlab (Mathworks; Natick, MA) scripts. Kinematic analyses and strength data were inherently standardized for body weight. Basic kinetic data, including gait speed and stride length, were computed.

For the gait analysis, the right and left legs were used to calculate kinematic measures during both walking conditions. The gait cycle was determined, beginning with the heel strike on the force plate and ending with the following heel strike, averaged, and normalized to 101 data points to allow comparisons between the two testing time points (pre- and post-treatment). The heel strike was determined in Visual3D (C-Motion) using a proprietary algorithm (i.e., force $>20 \mathrm{~N}$ ) [45]. Peak values were determined during the first $60 \%$ of stance, comparable to previous gait studies [46].

Regarding the DVJ, the stance phase, from initial contact with the force plate to the point at which the 
participant left the force plate, of each trial was determined, averaged, and normalized to 101 data points. Kinematic and kinetic waveforms were used to illustrate the data due to the immense amount of data extracted during the 3-D Motion analysis of the DVJ. The stance phase is depicted within the waveforms for the DVJ.

For dynamic postural control, composite scores were calculated for each participant by dividing the sum of the maximal reach in the anterior (A), posteromedial (PM) and posterolateral (PL) directions by three times the limb length (LL) of the individual, and then multiplying by $100\{[(\mathrm{~A}+\mathrm{PM}+\mathrm{PL}) /$ $(\mathrm{LL} \times 3)] \times 100\}$.

All data were entered and analyzed in SPSS. Descriptive statistics (means and standard deviations) on all biomechanics, strength and postural stability assessments were computed at pre- and post-treatment. Since this was a preliminary study targeted primarily at feasibility and initial evidence of effects, significance testing was not deemed appropriate. Effect sizes (Cohen's d) for all measures were computed to estimate the potential magnitude of treatment effects.

\section{Results}

Of the 17 initially enrolled JFM participants, 11 participants (Mean age $=16.00$ years, $S D=2.15 ; 100 \%$ females; 81.8 \% Caucasian) completed the FIT Teens intervention. Drop outs were due to schedule conflicts/transportation difficulties $(N=4)$ and need for referral for more intensive psychological/psychiatric care $(N=2)$. All 11 participants completed the dynamic postural stability and strength assessments (dataset available in Additional file 1), 10 completed the walking gait assessment, and eight completed the functional performance assessment (DVJ). One participant was not analyzed for walking gait or DVJ due to equipment difficulties, and two declined the DVJ due to discomfort or pain during the task.

\section{Walking gait}

Regarding gait velocity and stride length during selfselected gait and standardized gait, there did not appear to be any change in mean gait velocity from pre- to post-intervention; however, stride length appeared to show a small improvement ( $2 \mathrm{~cm}$ longer stride) from pre- to post-intervention (Table 2).

No other improvements in walking gait were observable in measured peak kinetic or kinematic variables.

\section{Knee and Hip strength}

Regarding knee and hip strength, participants demonstrated small-moderate improvement with increased peak torques in mean knee extension (small effect, Cohen's $\mathrm{d}=0.22-0.38$ ) and mean hip abduction
Table 2 Average velocity, stride length, and peak kinematic values for patients with JFM in the first $60 \%$ of stance during standardized gait $(n=10)$ and the land phase of the drop vertical jump $(n=8)$ before and after a neuromuscular training program

\begin{tabular}{|c|c|c|c|c|c|}
\hline & \multicolumn{2}{|l|}{ Pre } & \multicolumn{2}{|l|}{ Post } & \multirow[b]{2}{*}{$d$} \\
\hline & $\overline{\text { Mean }}$ & SD & $\overline{M e a n}$ & SD & \\
\hline \multicolumn{6}{|l|}{ Standardized Gait } \\
\hline Gait Velocity (m/s) & 0.82 & 0.02 & 0.83 & 0.04 & 0.32 \\
\hline Stride Length (m) & 0.85 & 0.04 & 0.88 & 0.04 & 0.75 \\
\hline Hip Flexion $\left({ }^{\circ}\right)$ & 26.74 & 3.07 & 27.56 & 3.46 & 0.25 \\
\hline Hip Adduction $\left({ }^{\circ}\right)$ & 10.31 & 0.94 & 10.29 & 2.11 & -0.01 \\
\hline Hip Internal Rotation $\left(^{\circ}\right)$ & 0.15 & 3.32 & -0.01 & 3.08 & -0.05 \\
\hline Knee Flexion $\left(^{\circ}\right)$ & -17.07 & 5.68 & -15.91 & 6.29 & 0.19 \\
\hline Knee Abduction $\left({ }^{\circ}\right)$ & -2.88 & 2.44 & -2.39 & 2.40 & 0.20 \\
\hline Knee Internal Rotation $\left(^{\circ}\right)$ & 4.38 & 2.32 & 4.59 & 3.15 & 0.08 \\
\hline Ankle Dorsiflexion $\left({ }^{\circ}\right)$ & 8.50 & 3.14 & 7.47 & 4.03 & -0.29 \\
\hline Ankle Eversion $\left({ }^{\circ}\right)$ & -5.80 & 1.83 & -6.20 & 1.92 & -0.21 \\
\hline \multicolumn{6}{|l|}{ Drop Vertical Jump } \\
\hline Hip Flexion $\left({ }^{\circ}\right)$ & 56.91 & 10.62 & 64.90 & 6.37 & 0.91 \\
\hline Hip Adduction $\left({ }^{\circ}\right)$ & 3.67 & 3.53 & 3.17 & 3.69 & -0.14 \\
\hline Hip Internal Rotation $\left(^{\circ}\right)$ & 4.96 & 4.86 & 4.38 & 4.29 & -0.13 \\
\hline Knee Flexion $\left(^{\circ}\right)$ & -82.10 & 9.41 & -82.32 & 5.32 & -0.03 \\
\hline Knee Abduction $\left(^{\circ}\right)$ & -9.70 & 5.74 & -9.82 & 5.34 & -0.02 \\
\hline Knee Internal Rotation $\left(^{\circ}\right)$ & 5.03 & 7.16 & 3.83 & 6.30 & -0.18 \\
\hline Ankle Dorsiflexion $\left({ }^{\circ}\right)$ & 30.68 & 4.06 & 29.13 & 4.12 & -0.38 \\
\hline Ankle Eversion $\left(^{\circ}\right)$ & 1.39 & 4.09 & 1.84 & 2.94 & 0.13 \\
\hline Trunk Flexion $\left({ }^{\circ}\right)$ & 16.89 & 6.95 & 23.62 & 7.04 & 0.96 \\
\hline
\end{tabular}

$\mathrm{m} / \mathrm{s}$ meters per second; ${ }^{\circ}$ degrees; bolded effect sizes denote medium-large effects

(moderate effect, Cohen's $\mathrm{d}=0.62-0.63$ ) from pre- to post-treatment (Table 4).

\section{Functional performance}

Regarding landing technique during the DVJ, maximum hip flexion angle and trunk flexion angle appeared to show strong increases after treatment (Cohen's $d=0.91$ and 0.96 respectively; Table 2 and Fig. 1). Regarding kinetic measures, mean internal hip extensor moment showed a large increase (Cohen's $\mathrm{d}=1.61$ ), while mean ankle eversion showed a moderate decrease Cohen's $d=-0.70$ ); see Table 3 and Fig. 2).

\section{Dynamic postural stability}

During the star excursion balance testing, dynamic postural control demonstrated moderate improvement bilaterally, as evidenced by increases in mean composite scores (Cohen's d = 0.61-0.67; Table 4). 


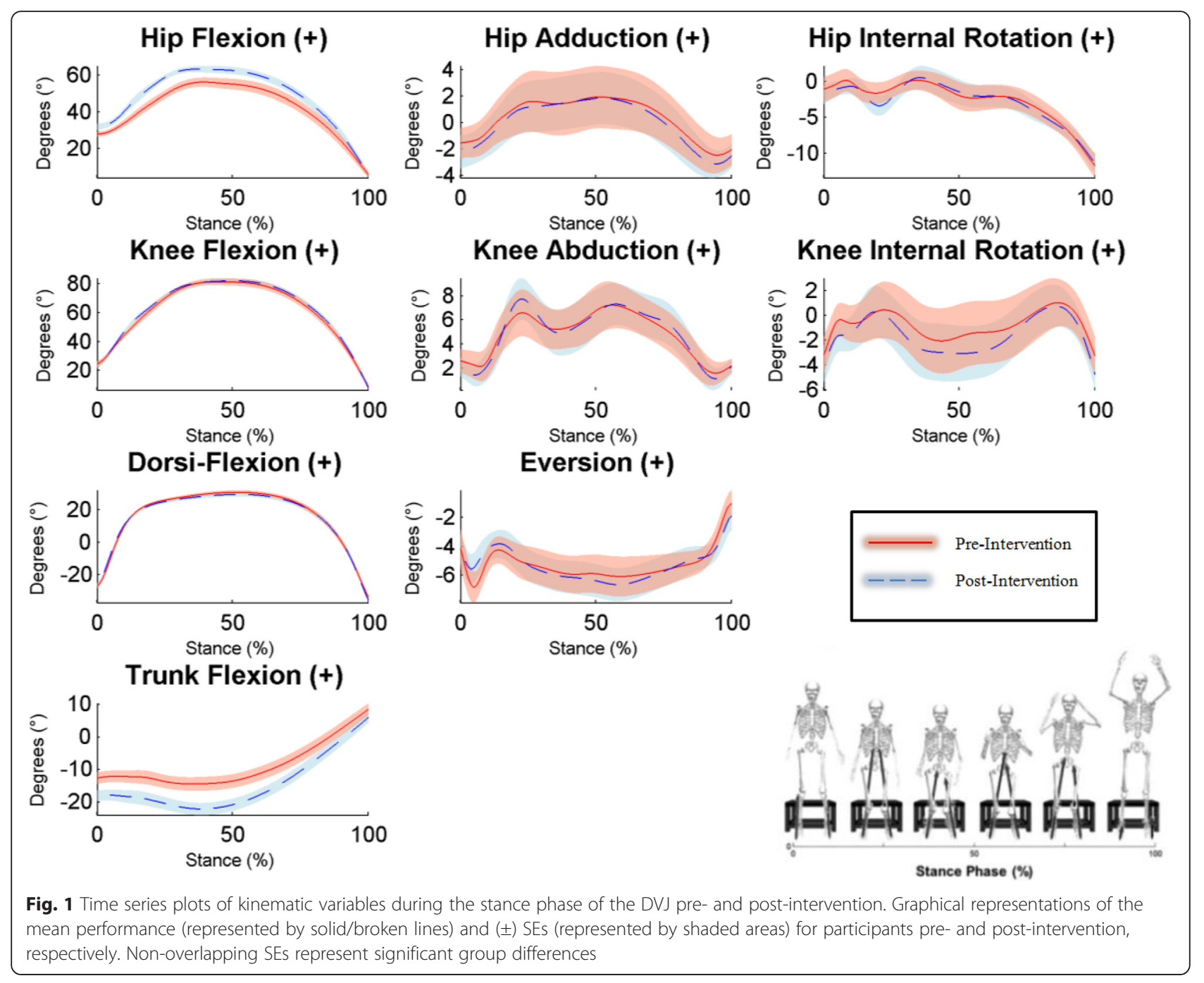

\section{Discussion}

The results of this study provide initial evidence for the utility of objective biomechanical assessments in measuring the effects of an 8-week integrated neuromuscular and CBT intervention on a range of measures of gait mechanics, knee and hip strength, functional performance and biomechanics, and dynamic postural stability in youth with JFM. Notably, participants demonstrated medium to large effect sizes in improved hip flexion and internal rotation gait mechanics, improved hip strength, improved hip and trunk flexion and ankle eversion during a functional landing task, and increased dynamic postural stability from pre- to post-treatment. These results not only demonstrate the promise of improved biomechanics in youth with JFM after the FIT Teens program, but this study also moves the field forward by utilizing a sophisticated assessment battery to examine improvements in movement mechanics in JFM. Few studies have examined biomechanics in fibromyalgia, and those that do tend to focus on one or two areas of muscle strength or control [47-49] rather than integrating multiple aspects of performance. In the context of the larger literature, the findings of the present study offer promise for improvement across multiple domains, and the sophisticated biomechanical assessments used in this study offer the opportunity to examine small changes which may translate into greater physical functioning.

Objective improvement in biomechanical performance may allow for safer and more stable execution of functional tasks of daily living, such as sit-tostanding, walking, stair climbing, etc., while minimizing risk of overexertion, stress, injury, and/or pain in individuals with chronic pain. The exercises in the FIT Teens program were specifically chosen to properly engage muscle groups that are essential to performing functional movements [43]. For instance, during the DVJ participants showed increases in hip flexion position and external hip flexion moment post-treatment, suggesting proper activation of the 
Table 3 Average (right and left leg) peak kinetic values for patients with JFM in the first $60 \%$ of stance during standardized gait $(n=10)$ and the land phase of the drop vertical jump $(n=8)$ before and after a neuromuscular training program

\begin{tabular}{|c|c|c|c|c|c|}
\hline & \multicolumn{2}{|l|}{ Pre } & \multicolumn{2}{|l|}{ Post } & \multirow[b]{2}{*}{$d$} \\
\hline & Mean & SD & $\overline{\text { Mean }}$ & SD & \\
\hline \multicolumn{6}{|l|}{ Standardized Gait } \\
\hline Hip Flexion (Nm) & 42.97 & 10.06 & 50.84 & 15.59 & 0.60 \\
\hline Hip Adduction (Nm) & 49.84 & 10.98 & 58.29 & 19.76 & 0.53 \\
\hline Hip Internal Rotation (Nm) & 5.26 & 1.72 & 7.64 & 3.15 & 0.94 \\
\hline Knee Flexion (Nm) & -24.12 & 12.61 & -26.16 & 14.35 & -0.15 \\
\hline Knee Abduction (Nm) & -3.90 & 2.23 & -4.62 & 2.31 & -0.32 \\
\hline Knee Internal Rotation (Nm) & 6.01 & 2.69 & 6.44 & 2.85 & 0.16 \\
\hline Ankle Dorsiflexion (Nm) & 45.21 & 11.20 & 48.10 & 11.24 & 0.26 \\
\hline Ankle Eversion (Nm) & -7.61 & 1.90 & -8.08 & 2.53 & -0.21 \\
\hline \multicolumn{6}{|l|}{ Drop Vertical Jump } \\
\hline Hip Flexion (Nm) & 63.29 & 7.80 & 81.51 & 14.07 & 1.61 \\
\hline Hip Adduction (Nm) & 36.66 & 22.13 & 32.22 & 23.04 & -0.20 \\
\hline Hip Internal Rotation (Nm) & 24.60 & 6.80 & 27.65 & 9.39 & 0.37 \\
\hline Knee Flexion (Nm) & 104.66 & 17.19 & 102.90 & 18.39 & -0.10 \\
\hline Knee Abduction (Nm) & 25.73 & 13.27 & 27.33 & 11.86 & 0.13 \\
\hline Knee Internal Rotation (Nm) & 5.50 & 2.94 & 6.68 & 2.59 & 0.43 \\
\hline Ankle Dorsiflexion (Nm) & 75.80 & 17.51 & 73.36 & 20.93 & -0.13 \\
\hline Ankle Eversion (Nm) & 12.48 & 4.36 & 9.87 & 2.92 & -0.70 \\
\hline
\end{tabular}

$\mathrm{Nm}$ newton-meters; bolded effect sizes denote medium-large effects

posterior chain and therefore, a safer landing mechanism (e.g., more effective deceleration and improved function during the landing phase of a dynamic movement). Likewise, the increased net hip internal rotator moment during walking gait signified enhanced recruitment of the hip external rotators, primarily the gluteus maximus and piriformis, which optimizes lower body alignment in such a way that maintains the knee position in line with the hips, as opposed to a valgus or "knock-knee" position which may leave a patient at higher risk of injury [50] and the development of anterior knee pain [51, 52]. Consequently, participants may also be able perform common activities of daily living that recruit the posterior chain and hip external rotators with improved mechanics and lower risk of injury, such as ascending/ descending stairs. Notably, adolescents with JFM and comorbid joint hypermobility were able to safely engage in the neuromuscular exercise training using modifications that ensured neutral joint positioning to protect against hyperextension [44]. The findings of improved joint strength and joint positioning may translate into the ability to safely engage in physical exercise and prevent joint injury for such patients.
Results of this study can also be interpreted with respect to known areas of biomechanical deficits in youth with JFM compared to their healthy peers. In a prior study, compared to healthy peers, adolescents with JFM were found to have significant deficits in knee and hip strength (particularly in hip abduction) and functional performance [22]. These specific areas appeared to show improvement after the FIT Teens intervention, particularly improved hip abduction strength and improved functional performance. Regarding hip abduction strength, there was a medium effect size for JFM participants improving hip strength, bringing them closer to healthy control subjects [22] by the end of the intervention. Improved trunk flexion during the DVJ post-treatment was also noted to look more similar to healthy control landing mechanics. These results suggest that through the course of the FIT Teens intervention, adolescents with JFM were able to progress towards normalized strength and biomechanics, which may translate into safer and more comfortable functional movement.

Information gathered from this study may be used to better design intervention protocols to target specific aspects of biomechanical functioning, as well as to inform health care providers and families about what aspects of biomechanics may be more difficult to correct for teens with JFM. Considering that the goal of this intervention was to improve integrated functional movement, by beginning with basic fundamental movement skills and confidence in movement, it is promising that postural stability and landing mechanics demonstrated improvement. Conversely, it is not surprising that relatively few of the biomechanical measures of walking gait demonstrated improvement given that there was less impairment in the walking gait of adolescents with JFM relative to healthy peers [22]. When considered in contrast to more complex functional tasks, interventionists may be able to focus less on walking gait (which may not require as much attention) in order to target more challenging movements, which showed improvement after the FIT Teens intervention.

\section{Limitations}

Although the results of this pilot study suggest that assessment measures were capable of detecting biomechanical changes after intervention, the sample size was small and there was no control group in this feasibility study. Further replication is needed to test whether improvements were statistically significant and could be more definitively attributed to the treatment. Another limitation of the current study is the brief nature of the intervention-eight weeks may not be a long enough 


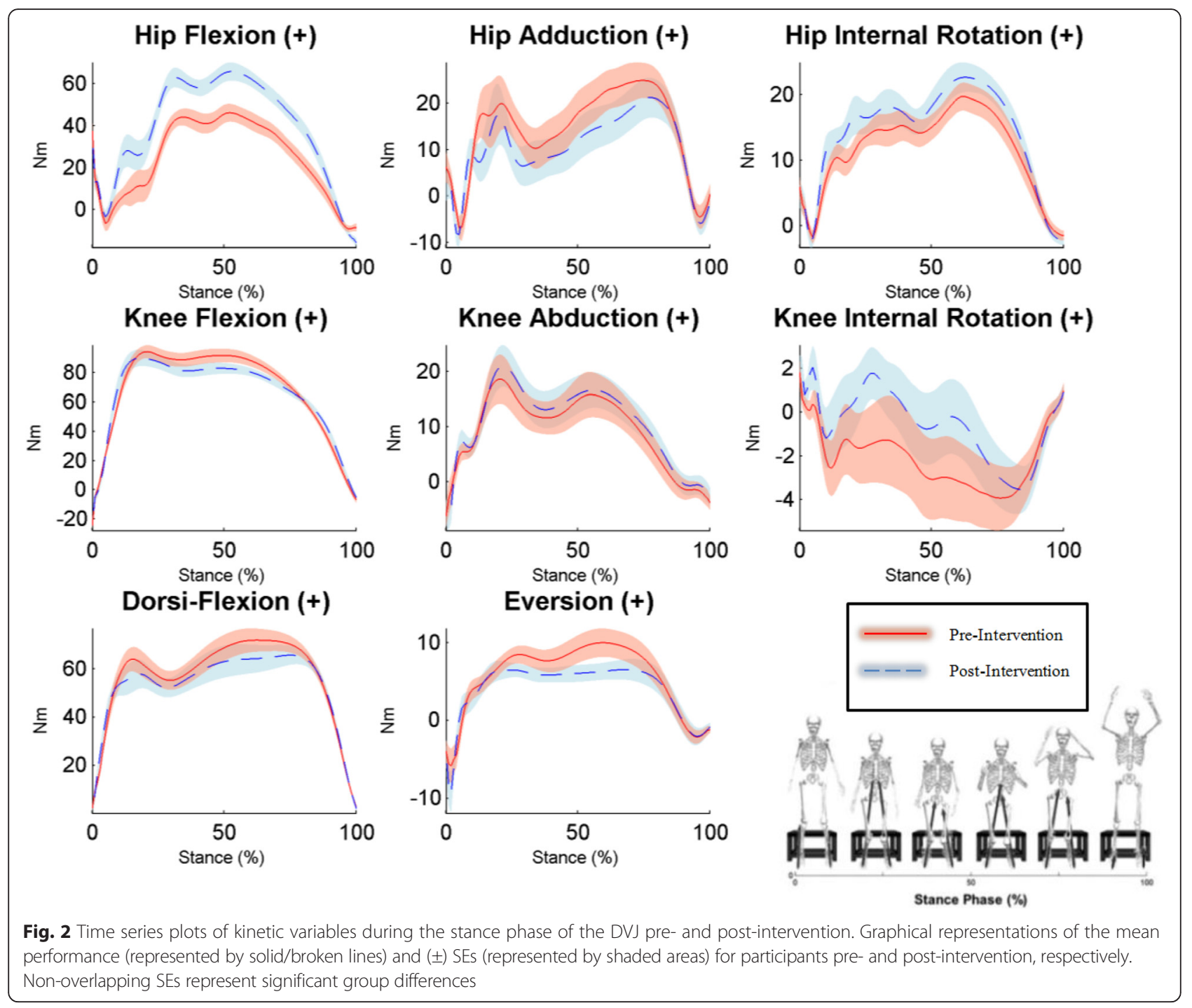

intervention to establish lasting changes in functional biomechanics. Still, there were signals that biomechanical changes were occurring in desirable direction for improved physical functioning.

\section{Future directions}

Future studies should examine how these objective changes in biomechanical performance may translate into improved daily functioning. It is unknown how much improvement in biomechanics is needed to enable one to participate in more physical exercise and activities of daily living. For example, use of actigraphy (using an accelerometer to assess time spent in vigorous and moderate activity) is one way of objectively monitoring physical activity to determine whether biomechanical changes correspond to actual increases in physical activity. Future studies would also benefit from use of a control group and, in order to determine whether changes after treatment are maintained over time, follow-up assessments should be conducted to assess biomechanics and physical functioning. Also, retention might be improved by offering sessions later in the afternoon/evening to prevent scheduling conflicts. In our current work, we are also including more formal screening for suicidal ideation which may suggest the need for more intensive mental health services to stabilize emotional status prior to participation. Finally, future studies may utilize larger sample sizes in order to examine how these objective measures of biomechanics and physical functioning relate to subjective reports of functional disability and confidence in movement.

\section{Conclusions}

Overall, results of this study offer initial evidence for the utility of biomechanical assessment measures in a complex pediatric chronic pain population. This sophisticated approach allowed for objective assessment of 
Table 4 Star excursion composite scores, isokinetic hip flexion and extension strength measures, and hip abduction strength measures for right and left sides in patients with JFM $(n=11)$ before and after a neuromuscular training program

\begin{tabular}{|c|c|c|c|c|c|}
\hline & \multicolumn{2}{|l|}{ Pre } & \multicolumn{2}{|l|}{ Post } & \multirow[b]{2}{*}{$d$} \\
\hline & Mean & SD & Mean & SD & \\
\hline \multicolumn{6}{|c|}{ Star Excursion (cm) } \\
\hline Right & 81.45 & 7.24 & 85.81 & 7.06 & 0.61 \\
\hline Left & 81.02 & 7.14 & 85.83 & 7.19 & 0.67 \\
\hline \multicolumn{6}{|c|}{ Knee Extension $300^{\circ} / \mathrm{s}(\mathrm{Nm} / \mathrm{kg})$} \\
\hline Right & 1.03 & 0.18 & 1.10 & 0.19 & 0.38 \\
\hline Left & 1.01 & 0.19 & 1.05 & 0.18 & 0.22 \\
\hline \multicolumn{6}{|c|}{ Knee Flexion $300^{\circ} / \mathrm{s}(\mathrm{Nm} / \mathrm{kg})$} \\
\hline Right & 0.71 & 0.12 & 0.74 & 0.15 & 0.22 \\
\hline Left & 0.72 & 0.13 & 0.73 & 0.18 & 0.06 \\
\hline \multicolumn{6}{|c|}{ Hip Abduction (Nm/kg) } \\
\hline Right & 0.73 & 0.28 & 0.90 & 0.26 & 0.63 \\
\hline Left & 0.72 & 0.23 & 0.91 & 0.37 & 0.62 \\
\hline
\end{tabular}

$\mathrm{cm}$ centimeters, $\mathrm{Nm} / \mathrm{kg}$ newton-meters per kilogram; bolded effect sizes denote medium-large effects

observable changes in biomechanical performance after an 8-week integrated CBT and neuromuscular training intervention for youth with JFM. Sophisticated measurement of biomechanics may offer enhanced capabilities to better design intervention protocols specifically targeting biomechanical deficits in patients with musculoskeletal pain for optimal outcomes. Results suggest that through the FIT Teens intervention, adolescents with JFM can potentially progress towards normalized strength and biomechanics, which may translate into improved movement confidence and engagement with physical exercise.

\section{Additional file}

Additional file 1: Balance and Strength database. (XLSX 16 kb)

\section{Abbreviations}

CBT, cognitive behavioral therapy; $\mathrm{cm}$, centimeter; DVJ, drop vertical jump task; FIT Teens, fibromyalgia integrative training for teens; JFM, juvenile fibromyalgia; Kg, kilogram; M, mean; Nm, newton-meter; SD, standard deviation

\section{Acknowledgments}

The authors would like to thank the patients and families who participated in the study for their time and effort.

\section{Funding}

This study was funded by the National Institute of Arthritis and Musculoskeletal and Skin Diseases (NIAMS)/NIH Grants K24AR056687 and R21AR063412 to the last author (SKZ).

\section{Availability of data and materials}

The dataset for strength and postural control is uploaded as a spreadsheet along with the manuscript. The motion capture system uses specialized software but is available from the authors upon request.

\section{Authors' contributions}

SKZ, GM, and TT made substantial contributions to the conception and design of the study, STT, ST, CD, MP, SS, and SEW made substantial contributions to the acquisition of data, and STT, ST, CD, MP, TT, SEW, GM, and SKZ were substantially involved in the analysis and interpretation of data. All authors were involved in drafting the manuscript or revising it critically, have given final approval of the current version, and are accountable for the accuracy and integrity of the work.

\section{Competing interests}

The authors declare that they have no competing interests.

\section{Consent for publication}

Not applicable.

\section{Ethics approval and consent to participate}

I confirm that this study has been approved by the appropriate ethics committee and that all participants and parents gave their informed consent and assent prior to their inclusion in the study.

\section{Author details}

${ }^{1}$ Department of Psychology, DePaul University, Chicago, IL, USA. ${ }^{2}$ Division of Sports Medicine, Sports Medicine Biodynamics Center and Human Performance Laboratory, Cincinnati Children's Hospital Medical Center, Cincinnati, OH, USA. ${ }^{3}$ Division of Behavioral Medicine and Clinical Psychology- MLC 7039, Cincinnati Children's Hospital Medical Center, 45229 Cincinnati, OH, USA. ${ }^{4}$ Department of Pediatrics, Emory University School of Medicine, Children's Healthcare of Atlanta, Aflac Cancer and Blood Disorders Center, Atlanta, GA, USA. 'Division of Rheumatology, Cincinnati Children's Hospital Medical Center, Cincinnati, OH, USA. ${ }^{6}$ Department of Pediatrics, University of Cincinnati, College of Medicine, Cincinnati, OH, USA. ${ }^{7}$ Department of Orthopaedic Surgery, University of Cincinnati, College of Medicine, Cincinnati, OH, USA. ${ }^{8}$ The Sports Health and Performance Institute, OSU Sports Medicine, Ohio State University Medical Center, Columbus, $\mathrm{OH}$, USA. ${ }^{9}$ The Micheli Center for Sports Injury Prevention, Boston, MA, USA.

Received: 13 April 2016 Accepted: 12 July 2016

Published online: 22 July 2016

\section{References}

1. Gedalia A, Press J, Klein M, Buskila D. Joint hypermobility and fibromyalgia in schoolchildren. Ann Rheum Dis. 1993;52:494-6.

2. Gerloni V, Ghirardini M, Fantini F. Assessment of nonarticular tenderness and prevalence of primary fibromyalgia syndrome in healthy Italian schoolchildren. Arthritis Rheum. 1998;41:1405.

3. Mikkelsson M, Salminen JJ, Kautiainen H. Non-specific musculoskeletal pain in preadolescents. Prevalence and 1-year persistence. Pain. 1997;73:29-35.

4. Sardini S, Ghirardini M, Betelemme L, Arpino C, Fatti F, Zanini F. Epidemiological study of a primary fibromyalgia in pediatric age. Minerva Pediatr. 1996:48:543-50.

5. Yunus MB, Masi AT. Juvenile primary fibromyalgia syndrome. A clinical study of thirty-three patients and matched normal controls. Arthritis \& Rheumatism. 1985;28:138-45.

6. Kashikar-Zuck S, Johnston M, Ting TV, Graham BT, Lynch-Jordan AM, Verkamp E, Passo M, Schikler KN, Hashkes PJ, Spalding S, et al. Relationship between school absenteeism and depressive symptoms among adolescents with juvenile fibromyalgia. J Pediatr Psychol. 2010;35:996-1004.

7. Kashikar-Zuck S, Allen R, Noll R, Graham T, Ho I, Swain N, Crain B, Mullen S. Anxiety and depressive symptoms in adolescents with juvenile fibromyalgia and their mothers. J Pain. 2005;6:31.

8. Kashikar-Zuck S, Lynch AM, Graham TB, Swain NF, Mullen SM, Noll RB. Social functioning and peer relationships of adolescents with juvenile fibromyalgia syndrome. Arthritis Rheum. 2007:57:474-80.

9. Kashikar-Zuck S, Parkins IS, Graham TB, Lynch AM, Passo M, Johnston M, Schikler KN, Hashkes PJ, Banez G, Richards MM. Anxiety, mood, and 
behavioral disorders among pediatric patients with juvenile fibromyalgia syndrome. Clin J Pain. 2008;24:620-6.

10. Kashikar-Zuck S, Vaught MH, Goldschneider KR, Graham TB, Miller JC. Depression, coping and functional disability in juvenile primary fibromyalgia syndrome. Journal of Pain. 2002;3:412-9.

11. Kashikar-Zuck S, Lynch AM, Slater S, Graham TB, Swain NF, Noll RB. Family factors, emotional functioning, and functional impairment in juvenile fibromyalgia syndrome. Arthritis Rheum. 2008:59:1392-8.

12. American Pain Society. Guideline for the management of fibromyalgia syndrome pain in adults and children. Glenview: American Pain Society: 2005

13. Eccleston C, Palermo TM, Williams AC, Lewandowski Holley A, Morley S, Fisher E, Law E. Psychological therapies for the management of chronic and recurrent pain in children and adolescents. Cochrane Database Syst Rev. 2014:5, CD003968.

14. Sherry DD, Brake L, Tress JL, Sherker J, Fash K, Ferry K, Weiss PF. The Treatment of Juvenile Fibromyalgia with an Intensive Physical and Psychosocial Program. J Pediatr. 2015;167:731-7.

15. Gowans $\mathrm{SE}$, deHueck $\mathrm{A}$. Effectiveness of exercise in management of fibromyalgia. Curr Opin Rheumatol. 2004;16:138-42.

16. Fontaine KR, Conn L, Clauw DJ. Effects of lifestyle physical activity in adults with fibromyalgia: results at follow-up. J Clin Rheumatol. 2011;17:64-8.

17. Kashikar-Zuck S, Flowers SR, Verkamp E, Ting TV, Lynch-Jordan AM, Graham TB, Passo M, Schikler KN, Hashkes PJ, Spalding S, et al. Actigraphy-based physical activity monitoring in adolescents with juvenile primary fibromyalgia syndrome. J Pain. 2010;11:885-93.

18. Kop WJ, Lyden A, Berlin AA, Ambrose K, Olsen C, Gracely RH, Williams DA, Clauw DJ. Ambulatory monitoring of physical activity and symptoms in fibromyalgia and chronic fatigue syndrome. Arthritis Rheum. 2005;52:296-303.

19. Korszun A, Young EA, Engleberg NC, Brucksch CB, Greden JF, Crofford LA. Use of actigraphy for monitoring sleep and activity levels in patients with fibromyalgia and depression. J Psychosom Res. 2002;52:439-43.

20. Jones K, King L, Mist S, Bennett R, Horak F. Postural control deficits in people with fibromyalgia: a pilot study. Arthritis Res Ther. 2011;13:R127.

21. Rabbitts JA, Holley AL, Karlson CW, Palermo TM. Bidirectiona associations between pain and physical activity in adolescents. Clin J Pain. 2014;30:251-8.

22. Sil S, Thomas S, DiCesare C, Strotman D, Ting TV, Myer G, Kashikar-Zuck S. Preliminary Evidence of Altered Biomechanics in Adolescents With Juvenile Fibromyalgia. Arthritis Care Res. 2015;67:102-11.

23. Hewett TE, Myer GD, Ford KR, Heidt RS, Colosimo AJ, McLean SG, Van den Bogert AJ, Paterno MV, Succop P. Biomechanical measures of neuromuscular control and valgus loading of the knee predict anterior cruciate ligament injury risk in female athletes A prospective study. Am J Sports Med. 2005:33:492-501

24. Paterno MV, Schmitt LC, Ford KR, Rauh MJ, Myer GD, Huang B, Hewett TE. Biomechanical measures during landing and postural stability predict second anterior cruciate ligament injury after anterior cruciate ligament reconstruction and return to sport. Am J Sports Med. 2010;38:1968-78.

25. Myer GD, Faigenbaum AD, Chu DA, Falkel J, Ford KR, Best TM, Hewett TE. Integrative training for children and adolescents: techniques and practices for reducing sports-related injuries and enhancing athletic performance. Phys Sportsmed. 2011;39:74-84.

26. Hewett TE, Ford KR, Myer GD. Anterior cruciate ligament injuries in female athletes: Part 2, a meta-analysis of neuromuscular interventions aimed at injury prevention. Am J Sports Med. 2006;34:490-8.

27. Nijs J, Kosek E, Van Oosterwijck J, Meeus M. Dysfunctional endogenous analgesia during exercise in patients with chronic pain: to exercise or not to exercise? Pain Physician. 2012;15:ES205-13.

28. Kashikar-Zuck S, Ting TV, Arnold LM, Bean J, Powers SW, Graham TB, Passo MH, Schikler KN, Hashkes PJ, Spalding S, et al. Cognitive behavioral therapy for the treatment of juvenile fibromyalgia: $A$ multisite, single-blind, randomized, controlled clinical trial. Arthritis Rheum. 2012;64:297-305.

29. Kashikar-Zuck S, Flowers SR, Strotman D, Sil S, Ting TV, Schikler KN. Physica activity monitoring in adolescents with juvenile fibromyalgia: findings from a clinical trial of cognitive-behavioral therapy. Arthritis Care Res (Hoboken). 2013;65:398-405

30. Kashikar-Zuck S, Tran ST, Barnett K, Bromberg MH, Strotman D, Sil S, Thomas SM, Joffe N, Ting TV, Williams SE, Myer GD. A Qualitative Examination of a
New Combined Cognitive-behavioral and Neuromuscular Training Intervention for Juvenile Fibromyalgia. The Clinical Journal of Pain. Publish Ahead of Print. (in Press).

31. Tran ST, Guite JW, Pantaleo A, Pfeiffer M, Myer GD, Sil S, Thomas SM, Ting TV, Williams SE, Edelheit B, et al. Preliminary outcomes of a cross-site cognitive-behavioral and neuromuscular integrative training intervention for juvenile fibromyalgia. Arthritis Care \& Research. (in Press).

32. Kashikar-Zuck S, Flowers SR, Claar RL, Guite JW, Logan DE, Lynch-Jordan AM, Palermo TM, Wilson AC. Clinical utility and validity of the Functional Disability Inventory (FDI) among a multicenter sample of youth with chronic pain. Pain. 2011;152:1600-7.

33. Ford KR, Myer GD, Melson PG, Darnell SC, Brunner HI, Hewett TE. Land-Jump Performance in Patients with Juvenile Idiopathic Arthritis (JIA): A Comparison to Matched Controls. Int J Rheumatol. 2009;2009: 478526.

34. Kwon JW, Son SM, Lee NK. Changes of kinematic parameters of lower extremities with gait speed: a 3D motion analysis study. J Phys Ther Sci. 2015;27:477.

35. Al-Obaidi S, Wall JC, Al-Yaqoub A, Al-Ghanim M. Basic gait parameters: A comparison of reference data for normal subjects 20 to 29 years of age from Kuwait and Scandinavia. J Rehabil Res Dev. 2003;40:361-6.

36. Öberg T, Karsznia A, Öberg K. Basic gait parameters: reference data for normal subjects, 10-79 years of age. J Rehabil Res Dev. 1993;30:210.

37. Myer G, Brent J, Ford K, Hewett T. A pilot study to determine the effect of trunk and hip focused neuromuscular training on hip and knee isokinetic strength. Br J Sports Med. 2008:42:614-9.

38. Brent IL, Myer GD, Ford KR, Paterno MV, Hewett TE. The effect of sex and age on isokinetic hip-abduction torques. J Sport Rehabil. 2013;22:41.

39. Ford KR, Myer GD, Hewett TE. Valgus knee motion during landing in high school female and male basketball players. Med Sci Sports Exerc. 2003;35: $1745-50$.

40. Aminaka N, Gribble PA. Patellar taping, patellofemoral pain syndrome, lower extremity kinematics, and dynamic postural control. J Athl Train. 2008:43:21-8

41. Gribble P, Hertel J, Denegar C. Chronic ankle instability and fatigue create proximal joint alterations during performance of the Star Excursion Balance Test. Int J Sports Med. 2007:28:236-42.

42. Gribble PA, Robinson RH, Hertel J, Denegar CR. The effects of gender and fatique on dynamic postural control. J Sport Rehabil. 2009;18:240.

43. Thomas SM, Sil S, Kashikar-Zuck S, Myer GD. Can Modified Neuromuscular Training Support the Treatment of Chronic Pain in Adolescents? Strength \& Conditioning Journal. 2013;35:12-26.

44. Ting TV, Hashkes PJ, Schikler K, Desai AM, Spalding S, Kashikar-Zuck S. The role of benign joint hypermobility in the pain experience in Juvenile Fibromyalgia: an observational study. Pediatric Rheumatol. 2012;10:16.

45. Stanhope S, Kepple T, McGuire D, Roman N. Kinematic-based technique for event time determination during gait. Med Biol Eng Comput. 1990; 28:355-60.

46. Ferber R, McClay Davis I, Williams D, Laughton C. A comparison of withinand between-day reliability of discrete 3D lower extremity variables in runners. J Orthop Res. 2002;20:1139-45.

47. Góes SM, Leite N, Shay BL, Homann D, Stefanello JM, Rodacki AL. Functional capacity, muscle strength and falls in women with fibromyalgia. Clinical Biomechanics. 2012;27:578-83.

48. Gerdle B, Grönlund C, Karlsson SJ, Holtermann A, Roeleveld K. Altered neuromuscular control mechanisms of the trapezius muscle in fibromyalgia. BMC Musculoskelet Disord. 2010;11:42.

49. Jiménez JMH, García-Molina VAA, Foulquie JMP, Fernández MD, Hermoso VMS. Spatial-temporal parameters of gait in women with fibromyalgia. Clin Rheumatol. 2009;28:595-8.

50. Hewett TE, Myer GD. The mechanistic connection between the trunk, hip, knee, and anterior cruciate ligament injury. Exerc Sport Sci Rev. 2011;39: 161-6.

51. Powers $\mathrm{CM}$. The influence of altered lower-extremity kinematics on patellofemoral joint dysfunction: a theoretical perspective. J Orthop Sports Phys Ther. 2003;33:639-46.

52. Myer GD, Ford KR, Di Stasi SL, Foss KDB, Micheli L, Hewett TE. High knee abduction moments are common risk factors for patellofemoral pain (PFP) and anterior cruciate ligament (ACL) injury in girls: Is PFP itself a predictor for subsequent ACL injury? Br J Sports Med. 2015;49:118-22. 